Reprod. Nutr. Dévelop., 1983, 23 (2 B), 443-451.

\title{
Relation entre insulinémie et adiposité dans deux lignées de poulets rendus maigres ou gras par sélection
}

\author{
J. SIMON, B. LECLERCO
}

Station de Recherches avicoles, I.N.R.A., Nouzilly, 37380 Monnaie, France.

Summary. Insulin-adiposity relationship in selected lean or fat lines of chickens.

Results obtained in two selected lines of chickens, lean (LL) or fat (FL), are reviewed. In both sexes, differences in adiposity between the lines set in at 2-4 weeks and were maximal at 9 weeks of age, at which time divergent selection was conducted. After 9 weeks, fattening of $F L$ and $L L$ females increased at a similar rate and that of FL and $L L$ males tended to plateau. Differences in adipocyte size appeared after 2 weeks of age. Food intake, metabolizable energy content of the diet, maintenance requirement and body temperature exhibited no differences between the lines. The fattening of both lines was modulated by the energy or protein content of the diet to approximately the same extent. Therefore, the difference between the lines was clearly of a metabolic order. In the $F_{3}$ generation, plasma glucose levels were similar at hatching and decreased in the FL in both the fed and fasted state at 2 weeks of age. In successive generations, plasma glucose levels were lower in the $\mathrm{FL}$ at progressively earlier ages : at hatching, then during the last third of embryonic development. However, this was not associated with higher plasma insulin levels in the FL during the posthatching period. Glucose tolerance in the FL was slightly accelerated from 4-6 weeks ; it then became normal at 8 weeks but was always associated with higher insulin release. Over the same age range, during refeeding following a fast (after a test meal or forced-feeding), plasma glucose levels were similar in both lines but, in contrast, insulin release was always largely amplified in the FL. Most of these features could be observed in the adult. An imbalance in the glucose-insulin relationship, analogous to that transiently observed in mutant preobese mammals, could therefore account for fattening in the FL. In chickens, however, this state does not ultimately result in true obesity. Several hypotheses could account for this pecularity.

\section{Introduction.}

La sélection constante des poulets pour des vitesses de croissance de plus en plus rapides s'est accompagnée d'une propension à un engraissement exagéré. Les moyens courants pour combattre cette tendance, taux protidiques élevés dans l'aliment (Blum, 1975) ou restrictions alimentaires (Simon et al., 1978) restent de toute façon peu efficaces, provoquent un gaspillage de protéines ou 
nécessitent des contrôles nombreux. Pour comprendre les mécanismes génétiques, nutritionnels ou hormonaux responsables, une sélection divergente sur l'engraissement a été entreprise chez des poulets de croissance rapide en utilisant comme critère le poids de graisses abdominales chez le mâle âgé de 9 semaines (Leclercq, Blum et Boyer, 1980). Le poids de graisses abdominales est en effet un bon indicateur de l'engraissement (Delpech et Ricard, 1965) et constitue un caractère hautement héritable (Ricard et Rouvier, 1967 et 1969 ; Leclercq et al., 1980). Deux lignées, maigre et grasse, présentant des vitesses de croissances similaires ont pu être isolées. Cette revue a pour objet de présenter une synthèse des modifications qui ont accompagné la divergence et de rechercher l'origine de cette dernière.

\section{Matériel et méthodes.}

Pour les données non publiées, les conditions expérimentales sont celles utilisées dans les publications précédentes (Leclercq et al., 1980; Simon et Leclercq, 1982).

\section{Résultats.}

Constitution des 2 lignées. - Le poids vif moyen chez les mâles oscille à 9 semaines autour de $2100 \mathrm{~g}$, de la population initiale à la $5^{e}$ génération (fig. 1). La baisse de performance observée à la $F_{6}$ est consécutive à un accident sanitaire (maladie de Marek). II est particulièrement intéressant de remarquer qu'aucune des 2 lignées n'apparaît systématiquement plus lourde ou plus légère. La liaison entre vitesse de croissance et adiposité n'est donc pas trop étroite ; elle peut être aisément rompue.

Les graisses abdominales exprimées en \% du poids vif divergent dès la première génération. La divergence maximale est atteinte à la $4^{e}$ génération. Le

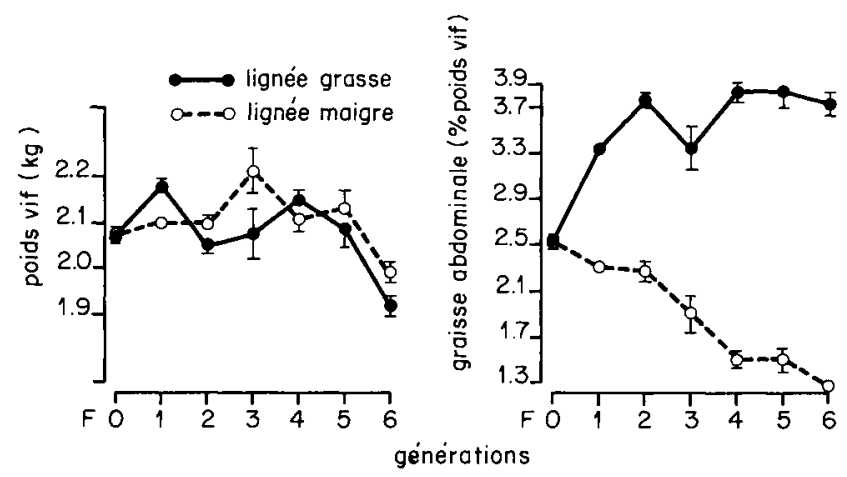

FIG. 1. - Evolution du poids vif et de l'engraissement (graisses abdominales en $\%$ du poids vif) au cours d'une sélection divergente pour l'engraissement chez des poulets âgés de 9 semaines. Les données sont exprimées par la moyenne \pm l'écart-typ̄e de la moyenne, $n=180-200$ là la génération $F_{3}, n=30$ ). 
léger amaigrissement constaté dans les 2 lignées à la $F_{6}$ paraît être la conséquence de l'accident sanitaire observé à cette génération. L'infléchissement de l'engraissement constaté dans la lignée grasse à la $F_{3}$ peut s'expliquer par le fait que la sélection à ce stade a été réalisée à 4 semaines et que les graisses abdominales n'ont été contrôlées à 9 semaines que sur un faible effectif (cf. légende de la figure 1). II est donc possible de sélectionner sur l'engraissement ; les progrès sont rapides et importants.

Etude temporelle de l'engraissement. - De façon à caractériser ces lignées, une étude de l'engraissement en fonction de l'âge a été réalisée à la $F_{4}$ dans les 2 sexes (Simon et Leclercq, 1982). Dès 2 semaines, le poids de graisses abdominales est significativement augmenté dans la lignée grasse. La différence est maximale à 9 semaines, âge où a eu lieu la sélection. Par la suite, ce dépôt augmente parallèlement chez les femelles maigres ou grasses jusqu'à au moins 25 semaines (soit 3 semaines après le début de ponte), alors qu'il tend à se stabiliser chez les mâles dès 9 semaines. Dans cette étude, l'engraissement des mâles n'avait pu être mesuré valablement au-delà de 17 semaines. Cela a été effectué à la $F_{5}$ à 40 semaines après reproduction au sol en parquet pedigree (unité comprenant 1 o et 5 ol. L'engraissement des mâles de la lignée grasse, bien qu'hétérogène et faible en valeur absolue, reste plus élevé que celui observé dans la lignée maigre ( $P<0,01$; tabl. 1). Chez les femelles à 40 semaines, il existe toujours une différence marquée (tabl. 1) ; l'écart n'est toutefois pas amplifié par rapport au stade 9 semaines (Simon et Leclercq, 1982).

\section{TABLEAU 1}

Engraissement et glycémie basale (16-17 h de jeûne) dans 2 lignées, maigre et grasse, à l'âge de 40 semaines (génération : $\left.F_{5}\right)\left({ }^{*}\right.$ )

\begin{tabular}{|c|c|c|c|c|}
\hline & \multicolumn{2}{|c|}{ Lignée grasse } & \multicolumn{2}{|c|}{ Lignée maigre } \\
\hline & Mâles & Femelles & Mâles & Femelles \\
\hline $\begin{array}{l}\text { Graisse abdominale ( } \% \text { poids vif) } \\
\text { Glycémie }(\mathrm{mg} / \mathrm{ml}) \ldots \ldots \ldots \ldots \ldots\end{array}$ & $\begin{array}{l}2,10 \pm 0,47 \\
1,93 \pm 0,02\end{array}$ & $\begin{array}{l}7,19 \pm 0,60 \\
1,87 \pm 0,02\end{array}$ & $\begin{array}{l}0,51 \pm 0,14 \\
2,16 \pm 0,03\end{array}$ & $\begin{array}{l}4,40 \pm 0,46 \\
2,00 \pm 0,03\end{array}$ \\
\hline
\end{tabular}

(*) Dans les 2 sexes les différences entre lignées sont significatives au minimum au seuil de $P<0,01$. Les données sont exprimées par la moyenne \pm l'écart-type de la moyenne, $n=15$.

L'évolution de la teneur en lipides corporels a été déterminée chez les mâles pour vérifier que la divergence ne concerne pas uniquement les graisses abdominales (Simon et Leclercq, 1982). Jusqu'à 2 semaines d'âge, les lignées ne diffèrent pas. L'engraissement apparaît plus marqué dans la lignée grasse à 4 semaines. La divergence est là encore maximale à 9 semaines, elle représente alors $50 \%$ d'augmentation dans la lignée grasse $19,5 \%$ de lipides totaux chez les maigres, $13 \%$ chez les gras). Par la suite, comme les graisses abdominales, les lipides corporels restent en plateau au moins jusqu'à 17 semaines. 
Une hypertrophie des adipocytes dans le dépôt entourant le gésier (dépôt qui fait partie intégrante de la masse utilisée pour la sélection) minime à 2 semaines devient manifeste à 4 semaines (Simon et Leclercq, 1982). Les différences ne sont jamais considérables. A 14 semaines, âge le plus avancé auquel la mesure a été pratiquée, le diamètre des adipocytes est augmenté de 20 à $25 \%$ dans la lignée grasse (chez les mâles, la taille moyenne \pm l'écart-type de la population sont $55 \pm 11 \mu \mathrm{m}$ dans la lignée grasse et de $44 \pm 9$ dans la lignée maigre). En volume, ces différences suffiraient à rendre compte de l'engraissement dans la lignée grasse. Etant donné qu'il reste toujours une réserve d'adipocytes de petite taille, inférieure à $10 \mu \mathrm{m}$, et sachant qu'il n'y a pas chez le poulet de dépôt vraiment bien délimité, il n'est pas possible d'exclure définitivement l'hypothèse selon laquelle une hyperplasie serait également présente dans la lignée grasse.

Les graisses abdominales et la taille des adipocytes divergent donc précocement ( 2 semaines). Une différence réelle dans l'engraissement ne s'installe qu'entre 2 et 4 semaines. Et si on retient une teneur de 20-25\% de lipides corporels comme seuil de l'obésité, la lignée grasse (au moins chez le mâle) n'évolue pas vers une obésité caractérisée.

Caractéristiques zootechniques et nutritionnelles des 2 lignées. - A la suite des diverses expériences de nutrition qui ont pu être réalisées, il n'apparaît pas de différences systématiques pour le poids vif ou la consommation d'aliment, mais l'efficacité alimentaire est, en toute logique, toujours moins bonne dans la lignée grasse. L'énergie ou les protéines du régime exercent leur effet classique et, semble-t-il, de façon proportionnée sur l'engraissement dans les 2 lignées (Touchburn, Simon et Leclercq, 1981). L'engraissement ne résulte donc pas d'une hyperphagie. Cela est particulièrement bien démontré en pair-feeding après un rationnement alimentaire d'intensité variable imposé entre 3 et 5 semaines, c'est-à-dire durant la période où la divergence s'installe (Leclercq et Saadoun, 1982). Alimentées à $45 \%$ de l'ad libitum, les 2 lignées présentent la même croissance et une rétention lipidique faible mais identique. La synthèse protéique globale ne serait donc pas intrinséquement réduite dans la lignée grasse. Pour les restrictions moins sévères : 60,75 et $90 \%$ de l'ad libitum, la lignée grasse présente un gain de poids plus faible mais à l'inverse une rétention lipidique toujours accrue. Il est donc clair que la propension de la lignée grasse à déposer plus de lipides ne résulte pas d'un appétit ou d'une consommation plus grande. Ces observations associées à des résultats préliminaires (Larbier et Leclercq, résultats non publiés) suggèrent également que la lignée grasse convertit une partie des protéines alimentaires en graisses corporelles.

Enfin, le besoin énergétique d'entretien estimé par régression pour des niveaux d'alimentation variable (ad libitum, 90, 75, 60 et $45 \%$ de l'ad libitum) est identique dans les 2 lignées (Leclercq et Saadoun, 1982). Les coefficients d'efficacité pour les rétentions énergétiques sous forme lipidique ou non lipidique sont également les mêmes. L'utilisation digestive du régime ne diffère pas non plus : pour un régime donné, l'énergie métabolisable mesurée est identique pour les 2 lignées (Leclercq et Saadoun, 1982).

En résumé l'adiposité de la lignée grasse n'est pas la conséquence d'une 
hyperphagie, d'un entretien faible ou d'une meilieure utilisation digestive de l'aliment. L'origine de la divergence apparaît donc uniquement d'ordre métabolique.

Aspects métaboliques. - Chez les mammifères, l'obésité s'accompagne et est même souvent précédée par une réduction de la température corporelle et de la thermogenèse (Yen, Fuller et Pearson, 1974; Trayhurn, Thurlby et James ; 1977 ; Godbole, York et Bloxham, 1978 ; Trayhurn, 1979). La température rectale a donc été mesurée dans les 2 lignées, à jeûn ou nourries, à 2 semaines. De l'état de jeûne à l'état nourri, la température augmente de $40^{\circ} 6$ à $41^{\circ} 1$ dans les 2 lignées (Touchburn et al., 1981). A 5 semaines, stade où les différences d'adiposité sont marquées, les températures sont à nouveau rigoureusement identiques. Le critère utilisé est certainement grossier et ne mesure pas les dépenses de thermogenèse. Il permet toutefois de conclure que dans une ambiance normale, une température corporelle variable n'est pas associée à des différences d'adiposité.

Une opposition marquée concerne la relation glycémie-insulinémie (Touchburn et al., 1981 ; Simon et Leclercq, 1982). Les premières mesures effectuées à la $F_{3}$ révèlent en effet qu'à 2 semaines, à jeûn ou nourrie, la lignée grasse présente une glycémie systématiquement plus faible et une insulinémie légèrement mais non significativement plus élevée. Ce déséquilibre a été retrouvé à jeûn dans 12 lots sur 15 au cours d'expériences réalisées entre 5 et 9 semaines. A l'âge adulte, la glycémie mesurée à jeûn reste plus faible chez les femelles (Leclercq et Simon, 1982), comme chez les mâles de la lignée grasse (tabl. 1). A l'éclosion, la glycémie des deux lignées est identique à la $F_{3}$ à 2 reprises mais apparaît significativement plus faible à la $F_{4}$ dans la lignée grasse. Il s'agit bien d'une apparition plus précoce de la divergence et non d'un artefact. La différence existe en effet à la $F_{4}$, que le prélèvement soit effectué immédiatement à la sortie de l'éclosoir, après une attente $(5 \mathrm{~h})$ à la température du laboratoire ou à $+4{ }^{\circ} \mathrm{C}$ (pendant $30 \mathrm{~min}$, une heure avant le prélèvement); l'insulinémie est alors identique entre les lignées (Simon et Leclercq, 1982). A partir de la $F_{4}$, la divergence apparaît dès le développement embryonnaire. Lorsque les mesures sont effectuées entre 15 et 19 jours d'incubation, la glycémie est significativement plus faible dans la lignée grasse à la $F_{5}$ de 16 à 18 jours alors que durant la même période elle augmentait de façon identique dans les 2 lignées à la $F_{4}$ (Simon et Leclercq, 1982).

La tolérance au glucose mesurée après surcharge orale $(2 \mathrm{~g} / \mathrm{kg}$ de poids vif) est faiblement accélérée dans la lignée grasse de 4 à 6 semaines (Touchburn et al., 1981) et à 17 semaines quand l'engraissement est modéré (Simon et Leclercq, 1982). Elle est normale à 8 ou 9 semaines. A tous les âges, la sécrétion d'insuline est augmentée dans la lignée grasse. Les mêmes mesures effectuées entre 5 et 8 semaines, après un repas libre ou ajusté $(25 \mathrm{~g})$ par gavage révèlent une évolution similaire pour la glycémie mais à nouveau une sécrétion d'insuline très amplifiée dans la lignée grasse. Ce déséquilibre dans la relation insulineglucose présente des analogies avec celui observé transitoirement chez les mutants mammifères pré-obèses (York, Steinke et Bray, 1972 ; Jeanrenaud, 1979 ; Dubuc, 1981). II constitue vraisemblablement le mécanisme responsable de l'engraissement de la lignée grasse. 
Entre 5 et 6 semaines d'âge, la triglycéridémie est plus élevée dans la lignée grasse à l'état nourri, en particulier lorsque le régime est pauvre en lipides. La différence avec la lignée maigre est amplifiée lorsque la mesure est effectuée 90 min après une injection de triton WR-1339 (Hermier et al., résultats non publiés). Cela suggère donc une sécrétion hépatique plus importante de VLDL dans la lignée grasse.

D'autres modifications ont accompagné la divergence pour l'engraissement. Le poids du vitellus est proportionnellement augmenté dans les œufs pondus par les femelles de la lignée grasse (Simon et Leclercq, 1982). La lignée grasse aurait donc tendance même à l'âge adulte à synthétiser et à déposer plus de lipoprotéines dans les follicules. Enfin, l'œuf contient très peu de glucose et ce glucose est presque totalement présent dans I'albumen (Romanoff, 1967). Le contenu en glucose (exprimé en valeur absolue ou par gramme d'albumen) est significativement plus faible dans la lignée grasse, ce qui reflète vraisemblablement la baisse de glycémie observée dans cette lignée même à l'âge adulte (tabl. 1, Leclercq et Simon, 1982).

\section{Discussion. Conclusion.}

A la naissance, il n'y a pas de différence d'engraissement entre lignées ; la divergence s'installe entre 2 et 4 semaines; elle est maximale vers 9 semaines, stade où la sélection a été réalisée. Toutefois, la lignée grasse (au moins chez le mâle) n'évolue pas vers une obésité manifeste. La déviation entre lignées est exclusivement d'origine métabolique : il n'y a pas d'hyperphagie dans la lignée grasse, pas de différence pour le poids, la température corporelle, le besoin d'entretien, l'utilisation digestive de l'aliment et les efficacités énergétiques pour les dépôts lipidiques et non lipidiques. Les modifications induites par la sélection semblent persister à l'état adulte entraînant dans la lignée grasse une vitellogenèse plus importante et une diminution de la concentration en glucose dans l'albumen. L'opposition la plus marquée concerne le déséquilibre glucose-insuline dans la lignée grasse en réponse au glucose ou après repas. Sans en connaître l'origine, on peut penser qu'il s'agit bien là du mécanisme responsable de la divergence. La sécrétion d'insuline dans la lignée maigre serait suffisante pour contrôler la glycémie et le transport du glucose dans les tissus dépendant de l'insuline. Le surcroît d'insuline dans la lignée grasse orienterait l'utilisation intracellulaire des nutriments vers la synthèse de lipides. Cette hormone stimule en effet les enzymes de la lipogenèse et la lipogenèse hépatique chez le poulet (Goodridge, 1973 ; Goodridge et Adelman, 1976 ; Tarlow et al., 1977 ; Joshi et Wakil, 1978 ; Pekala et al., 1978 ; Joshi et Aranda, 1979 a et b).

Les modifications observées peuvent apparaître discrètes en regard de la situation qui caractérise les mammifères obèses (cf. deux revues récentes : Jeanrenaud, 1979 ; Kahn, 1980). Elles présentent malgré tout des similitudes avec ce qui est connu chez l'homme dans certaines hyperlipémies (Tobey et al., 1981 ; Crapo, Reaven et Olefsky, 1981), chez le rat fa/fa préobèse au sevrage (York, Shargill et Godbole, 1981) et le rat obèse hypothalamique (Steffens, 1970).

La légère hypoglycémie apparaît de plus en plus tôt dans la lignée grasse : à 
l'éclosion $\left(F_{4}\right)$, et pendant le développement embryonnaire $\left(F_{5}\right)$. Elle n'est pas associée à une augmentation de l'insulinémie durant la première semaine de vie postnatale. Plusieurs hypothèses déjà formulées chez les mammifères (Jeanrenaud, 1979) pourraient expliquer cet équilibre particulier : un changement dans le contrôle nerveux ou hormonal augmenterait la sécrétion d'insuline en dépit d'une glycémie faible; la sensibilité des tissus à l'insuline serait accrue ; il y aurait un changement plus général dans le contrôle nerveux du métabolisme énergétique. On peut se demander pourquoi cette situation qui, comme il a été évoqué précédemment, ressemble à celle observée chez le jeune mammifère préobèse, n'évolue pas chez le poulet vers l'obésité caractérisée avec hyperphagie, hyperinsulinisme chronique et prise de poids exagérée. Plusieurs mécanismes pourraient rendre compte de cette particularité. Des gènes d'obésité existent bien chez le poulet mais n'étaient pas présents dans la population initiale. En dehors de l'obésité par autoimmunité thryoïdienne (Witebsky et al., 1969 ; Kite et al., 1969), cela n'a toutefois pas été décrit chez le poulet. L'homéostasie énergétique du poulet est contrôlée avec plus de précision que celle des mammifères; d'autres mécanismes se développent limitant l'engraissement dans la lignée grasse. Enfin, les récepteurs à l'insuline qui sont déjà rares chez le poulet comparativement au rat (Simon, Freychet et Rosselin, 1977 ; Kemmler et al., 1978 ; Simon, 1979) ne se raréfient pas davantage après les décharges d'insuline en réponse au repas (pas de " down regulation ") de telle sorte qu'il n'apparaît pas d'hyperinsulinisme dans la lignée grasse. En conséquence, le cycle autostimulant : hyperinsulinisme-résistance tissulaire, décrit chez les mammifères (Freychet, 1976 ; Kahn, 1980) serait absent chez le poulet.

$8^{e}$ Réunion du groupe Développement I.N.R.A., Tours, 12-13 mai 1982.

\section{Références}

BLUM J. C., 1975. Les apports azotés dans I'alimentation du poulet de chair. In : Les volailles de consommation, Nouzilly (France), Publ. I.N.R.A., 111-121.

CRAPO Ph. A., REAVEN G. M., OLEFSKY J. M., 1981. Hormonal and substrate responses to a standard meal in normal and hypertriglyceridemic subjects. Metabolism, 30, 331-334.

DELPECH P., RICARD F. H., 1965. Relation entre les dépôts adipeux viscéraux et les lipides corporels chez le poulet. Ann. Zootech., 14, 181-189.

DUBUC P. U., 1981. Non essential role of dietary factors in the development of diabetes in ob/ob mice. J. Nutr., 111, 1742-1748.

FREYCHET P., 1976. Interactions of polypeptide hormones with cell membrane specific receptors : studies with insulin and glucagon. Diabetologia, 12, 83-100.

GODBOLE V., YORK D. A., BLOXHAM D. P., 1978. Developmental changes in the fatty (fa/fa) rat: evidence for defective thermogenesis preceding the hyperlipogenesis and hyperinsulinaemia. Diabetologia, 15, 41-44.

GOODRIDGE A. G., 1973. Regulation of fatty acid synthesis in isolated hepatocytes prepared from the livers of neonatal chicks. J. biol. Chem., 248, 1924-1931.

GOODRIDGE A. G., ADELMAN T. G., 1976. Regulation of malic enzyme synthesis by insulin, triiodothyronine and glucagon in liver cells in culture. J. biol. Chem., 251, 3027-3032.

JEANRENAUD B., 1979. Insulin and obesity. Diabetologia, 17, 133-138. 
JOSHI V. C., WAKIL S. J., 1978. Hormonal regulation of hepatic tatty acid synthetase in chick embryo. Role of insulin. J. biol. Chem., 253, 2120-2125.

JOSHI V. C., ARANDA L. P., 1979 a. Hormonal regulation of the terminal enzyme of microsomal stearoyl coenzyme $A$ desaturase in cultured avian liver explants. Role of insulin. J. biol. Chem., 254, 11779-11782.

JOSHI V. C.. ARANDA L. P. $1979 \mathrm{~b}$. Hormonal regulation of fatty acid synthetase in cultured avian liver explants. J. biol. Chem., 254, 11783-11786.

KAHN C. R., 1980. Role of insulin receptors in insulin resistant states. Metabolism, 29, 455-466.

KEMMLER W., RENNER R., ZYNAMON A., HEPP K. D., 1978. Interactions between insulins and liver membrane receptors of guinea pig, calf and chicken. Exclusion of a species-specific insulin receptor. Biochim. biophys. Acta, 543, 349-356.

KITE J. H., WICK G., TWAROG B., WITEBSKY E., 1969. Spontaneous thyroiditis in the obese strain of chickens. II. Investigations on the development of the disease. J. Immunol., 103, $1331-1341$.

LECLERCO B., BLUM J. C., BOYER J. P., 1980. Selecting broilers for low or high abdominal fat : initial observations. Br. Poultry Sci, 21, 107-113.

LECLERCO B., SAADOUN A., 1982. Selecting broilers for low or high abdominal fat : comparison of energy metabolism of the lean and fat lines. Pouttry.Sci., 61, 1799-1803.

LECLERCO B., SIMON J., 1982. Selecting broilers for low or high abdominal fat : observations on the hens during the breeding period. Ann. Zootech., 31, 161-170.

PEKALA P. H., MEREDITH M. J., TARLOW D. M., LANE M. D., 1978. Multiple phosphorylation of acetyl-CoA carboxylase in chick liver cells. J. biol. Chem., 253, 5267-5269.

RICARD F. H., ROUVIER R., 1967. Etude de la composition anatomique du poulet. I. Variabilité de la répartition des différentes parties corporelles chez des coquelets "Bresse Pile ». Ann. Zootech., 16, 23-39.

RICARD F. H., ROUVIER R., 1969. Etude de la composition anatomique du poulet. II. Variabilité de la répartition des parties corporelles dans une souche de type Cornish. Ann. Génét. Sél. anim., 1, 151-165.

ROMANOFF A. L., 1967. Chemistry of the nonembryonic portions of the egg, 177-232. In ROMANOFF A. L. Biochemistry of the avian embryo, John Wiley and sons, New York, London, Sydney.

SIMON J., 1979. Effect of insulin on glucose oxidation and aminosisobutyric acid transport and binding of insulin in chicken thymocytes. Biochim. biophys. Acta, 585, 563-574.

SIMON J., FREYCHET P., ROSSELIN G., 1977. A study of insulin binding sites in the chicken tissues. Diabetologia, 13, 219-228.

SIMON J., LECLERCQ B., 1982. Longitudinal study of adiposity in chickens selected for high or low abdominal fat content. Further evidence of a glucose-insulin imbalance in the fat line. J. Nutr., 112, 1961-1973.

SIMON J., ZYBKO A., GUILLAUME J., BLUM J. C., 1978. Recherche d'une limitation de l'engraissement du poulet de chair par un léger rationnement alimentaire entre 6 et 8 semaines. Arch. Geflügelk., 42, 6-9.

STEFFENS A. B., 1970. Plasma insulin content in relation to blood glucose level and meal pattern in the normal and hypothalamic hyperphagic rat. Physiol. Behav., 5, 147-151.

TARLOW D. M., WATKINS P. A., REED R. E., MILLER R. S., ZWERGEL E. E., LANE M. D., 1977. Lipogenesis and synthesis and secretion of very low density lipoprotein by avian liver cells in non proliferating monolayer culture. Hormonal effects. J. Cell Biol., 73, 332-353.

TOBEY T. A., GREENFIELD M., KRAEMER F., REAVEN G. M., 1981. Relationship between insulin resistance, insulin secretion, very low density lipoprotein kinetics and plasma triglycerides levels in normotriglyceridemic man. Metabolism, 30, 331-334.

TOUCHBURN S., SIMON J., LECLERCO B., 1981. Evidence of a glucose-insulin imbalance and effect of dietary protein and energy level in chickens selected for high abdominal fat content. J. Nutr., 111, 325-335.

TRAYHURN P., 1979. Thermoregulation in the diabetic-obese $(\mathrm{db} / \mathrm{db})$ mouse. The role of nonshivering thermogenesis in energy balance. Pflügers Arch., 380, 227-232.

TRAYHURN P., THURLBY P. L., JAMES W. P. T., 1977. Thermogenic defect in pre-obese ob/ob mice. Nature, 266, 60-61. 
WITEBSKY E., KITE J. H., WICK G., COLE R. K., 1969. Spontaneous thyroiditis in the obese strain of chickens I. Demonstration of circulating autoantibodies. J. Immunol., 103, 709-715.

YEN T. T., FULLER R. W., PEARSON D. V., 1974. The response of " obese " (ob/ob) and " diabetic" $(\mathrm{db} / \mathrm{db})$ mice to treatments that influence body temperature. Comp. Biochem. Physiol., 49A, 377-385.

YORK D. A., SHARGILL N. S., GODBOLE V., 1981. Serum insulin and lipogenesis in the suckling "fatty" fa/fa rat. Diabetologia, 21, 143-148.

YORK D. A., STEINKE J., BRAY G. A., 1972. Hyperinsulinemia and insulin resistance in genetically obese rats. Metabolism, 21, 277-284. 